\title{
Glucagon-Like Peptide-1: A Focus on Neurodegenerative Diseases
}

\author{
Maddalena Grieco', Alessandra Giorgi', Maria Cristina Gentile², Maria d'Erme', \\ Susanna Morano ${ }^{2}$, Bruno Maras ${ }^{1}$ and Tiziana Filardi ${ }^{2 *}$ \\ ${ }^{1}$ Department of Biochemical Sciences, Faculty of Pharmacy and Medicine, Sapienza University of Rome, Rome, Italy, \\ ${ }^{2}$ Department of Experimental Medicine, Faculty of Medicine and Dentistry, Sapienza University of Rome, Rome, Italy
}

\section{OPEN ACCESS}

Edited by:

Mara Dierssen,

Centre for Genomic Regulation

(CRG), Spain

Reviewed by:

Alberto Granzotto,

Center for Research on Aging Sciences and Translational Medicine

Center (CeSI-MeT), Italy

Christian Hölscher,

Lancaster University, United Kingdom

*Correspondence:

Tiziana Filardi

tiziana.filardi@uniroma1.it

Specialty section:

This article was submitted to

Neurodegeneration,

a section of the journal

Frontiers in Neuroscience

Received: 14 June 2019 Accepted: 02 October 2019 Published: 18 October 2019

Citation:

Grieco M, Giorgi A, Gentile MC, d'Erme M, Morano S, Maras B and

Filardi T (2019) Glucagon-Like

Peptide-1: A Focus on

Neurodegenerative Diseases.

Front. Neurosci. 13:1112.

doi: 10.3389/fnins.2019.01112
Diabetes mellitus is one of the major risk factors for cognitive dysfunction. The pathogenesis of brain impairment caused by chronic hyperglycemia is complex and includes mitochondrial dysfunction, neuroinflammation, neurotransmitters' alteration, and vascular disease, which lead to cognitive impairment, neurodegeneration, loss of synaptic plasticity, brain aging, and dementia. Glucagon-like peptide-1 (GLP-1), a gut released hormone, is attracting attention as a possible link between metabolic and brain impairment. Several studies have shown the influence of GPL-1 on neuronal functions such as thermogenesis, blood pressure control, neurogenesis, neurodegeneration, retinal repair, and energy homeostasis. Moreover, modulation of GLP-1 activity can influence amyloid $\beta$ peptide aggregation in Alzheimer's disease (AD) and dopamine (DA) levels in Parkinson's disease (PD). GLP-1 receptor agonists (GLP-1RAs) showed beneficial actions on brain ischemia in animal models, such as the reduction of cerebral infarct area and the improvement of neurological deficit, acting mainly through inhibition of oxidative stress, inflammation, and apoptosis. They might also exert a beneficial effect on the cognitive impairment induced by diabetes or obesity improving learning and memory by modulating synaptic plasticity. Moreover, GLP-1RAs reduced hippocampal neurodegeneration. Besides this, there are growing evidences on neuroprotective effects of these agonists in animal models of neurodegenerative diseases, regardless of diabetes. In PD animal models, GPL-1RAs were able to protect motor activity and dopaminergic neurons whereas in $A D$ models, they seemed to improve nearly all neuropathological features and cognitive functions. Although further clinical studies of GPL-1RAs in humans are needed, they seem to be a promising therapy for diabetes-associated cognitive decline.

Keywords: glucagon-like peptide-1, GLP-1 receptor agonists, Parkinson's disease, Alzheimer's disease, neurodegenerative diseases, type 2 diabetes

\section{INTRODUCTION}

The concern for neurodegeneration, a worldwide expanding set of diseases, stimulated the research on risk factors related to the lifestyle of the population, leading to interesting findings on the association between dysmetabolism and brain impairment. In this perspective, gut/brain axis and altered insulin release and response seem to be the main actors in establishing the pathological metabolic set up for the development of neurodegenerative diseases. Indeed, insulin works as prosurvival neurotrophic factor with its receptor widespread in cognitive areas as hippocampus and in dopaminergic system (Haas et al., 2016; Fiory et al., 2019). 
In the last years particular attention has been devoted to unravel the role of glucagon-like peptide-1 (GLP-1), a gut released hormone that not only is one of the major components of the gut/brain axis, but it is also able to protect pancreatic beta-cells from apoptosis and to induce insulin secretion (Cabou and Burcelin, 2011).

Glucagon-like peptide- 1 is an endogenous peptide hormone released by intestinal L-cells in response to meal. Gene expression generates pro-glucagon (PG), which is processed by prohormone convertases (PC1/3) to release the GLP-1 (1-37) peptide precursor (Sandoval and D'Alessio, 2015). Proteolytic cleavage and amidation of the precursor protein GLP-1(137) generate two GLP-1 active forms with the same biological activity namely GLP-1 (7-37) and the amidated GLP-1 (7-36). GLP-1 is degraded by a dipeptidyl-peptidase IV (DPP IV), a serine aminopeptidase expressed in the different organ, such as liver, pancreas, gut, and brain (Hopsu-Havu and Glenner, 1966; Smith et al., 2019). GLP-1 stimulates insulin secretion from the pancreatic beta-cells under hyperglycemic conditions and reduces glucagon secretion from the alfa-cells recovering insulin sensitivity and enhancing glycemic homeostasis (Meloni et al., 2013; Katsurada and Yada, 2016).

Glucagon-like peptide-1 signal transduction is mediated by GLP-1 receptor (GLP-1R), a G-protein coupled receptor, leading to cyclic adenosine monophosphate (cAMP) dependent activation of protein kinase A (PKA) and of cAMP-regulated guanine nucleotide exchange factor (Epac). The activation of Epac and PKA potentiates in a synergistic way the insulin release from the beta-pancreatic cells through phosporylation of the SNARE-associated protein Snapin and activation of L-type voltage gated calcium channels (Song et al., 2011).

It is noteworthy that GPL-1R may operate signal transduction even by activating the PI3K/AKT axis as observed in GLP1 protection against apoptosis with the regulation of CREB and protein survival factors like $\mathrm{Bcl}-2$ and $\mathrm{Bcl}-\mathrm{XL}$, through the action of $\beta$-arrestin- 1 and the phosphorylation of ERK1/2. Furthermore, the activation of the PI3K/AKT axis can induce the inhibition of specific caspases and of $N F-\kappa B$, with the resulting inhibition of the release of pro-inflammatory cytokines (Farilla et al., 2003; Athauda and Foltynie, 2016; Tramutola et al., 2017; Yang et al., 2018).

Plasticity of GLP-1 action at molecular level is mirrored also in different tissues like the cardiac compartment and the brain. GLP1 plays a pivotal role preventing cardiovascular disorders, which makes GLP-1 and its analogs a great resource in the treatment of these diseases (Pozo et al., 2019). GLP-1 is also involved in the reduction of the oxidative stress, in the regulation of autophagy, and in the modulation of central nervous system (CNS) pathways with protective functions and elicitation of anti-inflammatory signaling (Li et al., 2009).

\section{MULTIFUNCTIONAL ROLE OF GLP-1}

Glucagon-like peptide-1 is produced at neuronal level of the solitary tract within the brainstem. In addition, this peptide, released from the gut, activates the GLP-1R located on vagal sensory neurons that constitute the hepato-portal glucose sensor, communicating with brainstem neurons, extending its action to different brain regions. Several studies have shown the influence of GLP-1 on neuronal function such as thermogenesis, blood pressure control, neurogenesis, neurodegeneration, retinal repair, and energy homeostasis (Katsurada and Yada, 2016). Since GLP-1Rs are expressed in different brain regions, GLP1 behaves as a neuropeptide, involved in different peculiar effects including the control of satiety, water intake, and stress reaction (van Dijk and Thiele, 1999; Meier et al., 2002). Kinzig et al. (2003) reported that GLP-1-stimulated brain GLP-1Rs are mediator of multiple stress responses. GLP-1 administration directly into the rat brain increases anxiety level, associated with a higher production of stress-activated hormones ACTH and corticosterone, demonstrating that GLP-1 is able to stimulate at the same time a response by both amygdala and by the paraventricular nucleus of the hypothalamus (Kinzig et al., 2003). The increase of GLP-1 in the circulation could reach the brain and regulate food intake (Ruttimann et al., 2009). Recent studies showed that GLP-1 in combination with dexamethasone (GLP1/Dexa) can decrease food intake and lower body weight in obese mice without inducing mood or memory deficits (DecarieSpain et al., 2019). In type 2 diabetes (T2D), alteration of insulin sensitivity and disturbances of neurogenesis are correlated with a reduction in GLP-1 levels in response to food, and its signaling activity (Nauck et al., 2011). Recently, several groups reported that GLP-1 contributes to the regulation of neurologic and cognitive functions (Muscogiuri et al., 2017). Indeed, GLP1 is also involved in the control of the synaptic plasticity and in some forms of neuroprotection and thus has a regulative role in various signaling pathways associated with learning, memory, and other synaptic function (Gault and Holscher, 2008; Yildirim Simsir et al., 2018).

\section{GLUCAGON-LIKE PEPTIDE-1 RECEPTOR AGONISTS (GLP-1RAS) AS NEUROPROTECTIVE AGENTS IN DIABETES-ASSOCIATED COGNITIVE IMPAIRMENT}

Type 2 diabetes is a chronic disease with an increasing global prevalence. Besides the well-known micro- and macro-vascular complications, cognitive decline is thought to be an emerging consequence of diabetes (Koekkoek et al., 2015).

Over the last decade GLP-1RAs have emerged as effective glucose-lowering drugs. Exenatide was the first GLP-1RA approved for the treatment of T2D. It is a synthetic form of exendin-4, a natural GLP-1-like peptide extracted from the saliva of the lizard Heloderma suspectum. Exenatide shares 53\% homology with native GLP-1. Exenatide has a half-life of $2.4 \mathrm{~h}$, whereas GLP-1 has a half-life of $2 \mathrm{~min}$. Lixisenatide is based on the structure of exendin- 4 and has a half-life of $3 \mathrm{~h}$. Liraglutide was the first GLP-1RA deriving from native GLP-1, sharing 97\% homology and with a half-life of $13 \mathrm{~h}$ (Aroda, 2018). Semaglutide, a modified form of liraglutide has a half-life of $\sim 7$ days due 
to a 5.6 times higher affinity with albumin than liraglutide (Gomez-Peralta and Abreu, 2019).

Several studies have investigated the neuroprotective actions of GLP-1RAs in animal models of diabetes. Many of them have focused on the effects of GLP-1RAs on cerebral ischemia/reperfusion injury. In diabetic rats with cerebral ischemia/reperfusion damage caused by middle cerebral artery occlusion, recombinant GLP-1 improved neurological deficit and reduced cerebral infarct area, mainly through the inhibition of oxidative stress and apoptosis (Fang et al., 2018). GLP-1RAs exert favorable effects, such as the reduction of cognitive impairment induced by diabetes or obesity.

Indeed, it has been observed that peripheral administration of lixisenatide for 40 days (50 nmol/ $\mathrm{kg}$ bw, twice-daily) in high-fat fed mice with established obesity, insulin resistance, and impaired cognition resulted in marked improvement in recognition memory, which was associated with up-regulation of hippocampal expression of neurotrophic tyrosine kinase receptor type 2 and mammalian target of rapamycin (mTOR) genes involved in modulating synaptic plasticity and longterm potentiation. Lixisenatide treatment promoted also hippocampal progenitor cells proliferation and increased immature neurons in the hippocampal dentate gyrus (Lennox et al., 2014). Liraglutide showed effects against hippocampal neurodegeneration induced by streptozotocin (STZ), an animal model of diabetes and neurodegeneration associated with cognitive decline. In particular, liraglutide improved learning and memory, and reduced hippocampal neuronal death (Palleria et al., 2017). In addition, in a STZ-induced mouse model of diabetes, pre-treatment with liraglutide contrasted neuronal and synaptic damage in the hippocampal CA1 region (Kong et al., 2018).

Notably, neuroprotective activity of GLP-1RAs seem not to be entirely related to glycemia normalization. Indeed, there is growing evidence about neuroprotective effects of GLP-1RAs in animal models of neurodegenerative diseases, regardless of diabetes. Liraglutide reduced infarct size in the brain of diabetic and non-diabetic rats but decreased neurologic deficits only in non-diabetic rats, suggesting that the GLP-1 RAs effects on cognitive function are not associated with diabetes and glycemia normalization. Indeed, both liraglutide and metformin, a glucose lowering agent acting via AMP-activated protein kinase-dependent pathways, induced euglycaemia in diabetic rats, but only liraglutide treatment reduced ischemic brain damage (Filchenko et al., 2018).

\section{GLP-1RAs AS NEUROPROTECTIVE AGENTS IN NEURODEGENERATIVE DISEASES}

Considering the beneficial effects of GLP-1RAs on neuropathological features it is conceivable a link between T2D and neurodegenerative disease such as Parkinson's disease (PD) and Alzheimer's Disease (AD). Neurodegenerative diseases have a considerable physical, psychological, social, and economical impact, both on affected people and on their careers, families, and society in general (World Health Organization, 2017).

\section{Parkinson's Disease}

Parkinson's disease is a progressive nervous system disorder whose etiology remains still unclear, although genetic and environmental factors seem to be involved. PD's clinical features include resting tremor, rigid muscles, slowed movement (bradykinesia), postural instability, and loss of purposeful movement (Kalia and Lang, 2015). Pathological features are characterized by neurons impairment of substantia nigra pars compacta with concomitant formation of intracellular Lewy bodies and loss of dopaminergic neurons. Lewy bodies are abnormal aggregates of $\alpha$-synuclein protein, which is involved in dopamine (DA) metabolism and function. Dopaminergic neurons dysfunction and death by apoptosis or autophagy are also associated with mitochondrial activity alteration, oxidative stress, altered protein handling, and inflammatory condition (Olanow and Tatton, 1999). Rare dominant form of PD in familial and sporadic cases is associated to point mutations, duplications, and triplications in the $\alpha$-synuclein gene (Lesage and Brice, 2009).

In different preclinical models of PD, GLP-1RAs showed neuroprotective effects, influencing motor activity, dopaminergic neurons, cortical activity, and energy utilization in the brain. Harkavyi et al. tested the efficacy of exendin-4 in rat models of PD treated with 6-hydroxydopamine (6-OHDA) and lipopolysaccaride (LPS) (Harkavyi et al., 2008). They observed that in striatal tissue DA concentrations were markedly higher in 6-OHDA/LPS + exendin-4 treated rats with respect to 6OHDA/LPS + vehicle groups. This effect was associated with an increase in the tyrosine hydroxylase enzyme involved in the production of L-dopa, a DA precursor. In the same PD rat model exendin-4 was able to promote adult neurogenesis in vitro e in vivo, normalizing $\mathrm{DA}$ imbalance, showing an increase in tyrosine hydroxylase- and vesicular monoamine transporter 2-positive neurons in the substantia nigra (Bertilsson et al., 2008). Other authors observed that the administration of exendin-4, liraglutide, and lixisenatide in the same mouse model prevented both motor dysfunction and tyrosine hydroxylase levels reduction in the substantia nigra and basal ganglia. Furthermore, liraglutide and lixisenatide induced a marked increase in anti-apoptotic pathways compared to exendin-4 (Liu et al., 2015).

Recently, the long-term administration of liraglutide was found to rescue dopaminergic neuronal loss and motor impairment also in diabetic $\mathrm{db} / \mathrm{db}$ mice, an established model of diabetes, with a mutation in the gene encoding the leptin receptor (Ma et al., 2019), suggesting that long-term injection of liraglutide might prevent motor function impairment and PD development also in patients with T2D. In rotenone-induced PD model, liraglutide together with sitagliptin, a DPP IV inhibitor, increased striatal DA and tyrosine hydroxylase protein levels, reduced neuroinflammation, and reversed neuronal loss (Badawi et al., 2017). Liraglutide was also able to attenuate dyskinesia, a serious complication of long-term therapy with L-dopa (Badawi et al., 2019). 
In the MPTP mouse model of PD, semaglutide improved most of neuropathological features of $\mathrm{PD}$, reversing motor impairment, inducing the increase of tyrosine hydroxylase levels, and attenuating neuroinflammation and apoptosis in the substantia nigra and striatum (Zhang et al., 2018). A reduction in $\alpha$-synuclein aggregation occurred after this treatment, not observed with other GLP-1RAs (Zhang et al., 2019), highlighting semaglutide as an effective treatment for PD.

Preliminary clinical studies were performed with subcutaneous injections of exenatide in PD patients. Athauda et al. (2017) reported the results of the first randomized, doubleblind, placebo-controlled trial in 62 patients affected by moderate PD. Patients were randomly assigned to receive subcutaneous injections of exenatide $2 \mathrm{mg}$ once-weekly (n.32) or placebo (n.30) for 48 weeks. Exenatide had positive and sustained effects (12 weeks after exposure) on clinically assessed motor function. A post hoc analysis indicated that even non-motor symptoms, such as clinically evaluated mood and emotional well-being, improved in patients treated with exenatide although these beneficial effects did not last after interruption (Athauda et al., 2018). Patients treated with exenatide had significantly higher tyrosine phosphorylation of insulin receptor (IR) substrate 1 and higher expression of total Akt and phosphorylated mTOR than placebo-treated patients providing a possible insulin-based molecular mechanism explanation for the results observed in clinical trial (Athauda et al., 2019).

\section{Alzheimer's Disease}

Dementia is a chronic disease, which affects memory, other cognitive abilities and behavior. It is estimated that approximately 50 million people worldwide have dementia. Currently, it is the 7 th leading cause of death and it is one of the major causes of disability worldwide. Pre-diabetic risk factors, obesity, and metabolic syndrome can promote cognitive dysfunction. AD is the most common form of dementia, contributing to 60$70 \%$ of cases. The main neuropathological features of $\mathrm{AD}$ are neurofibrillary tangles, formed by hyperphosphorylated tau proteins, which aggregate into oligomers, and the amyloid plaques, formed by aggregated $\beta$-amyloid peptides (A $\beta$ ) (Calsolaro and Edison, 2015).

Increasing evidence suggests a link between T2D and AD. In particular, these conditions might share defects in insulin signaling. Interestingly, in a mouse model of genetically induced AD-like neuropathology (3xTg-AD mice) peripheral glucose intolerance was observed. Treatment with pioglitazone, a glucose lowering drug, greatly improved cognitive impairment of these mice confirming the neurotrophic role of insulin (Masciopinto et al., 2012). In the same model, high-fat diet further potentiated glucose intolerance and enhanced neuropathological features of $\mathrm{AD}$ and memory deficits. Insulin adoption reversed the negative effect of high-fat diet, interrupting the vicious cycle between diabetes and $\mathrm{AD}$ (Vandal et al., 2014). Both studies highlighted the neurotrophic role of insulin in brain.

Conversely, hyperinsulinemia induced by peripheral administration of insulin increased tau phosphorylation by in C57BL/6 mice (Freude et al., 2005).
Aggregated $\beta$-amyloid peptide oligomers induced reduction of IRs activity due to the phosphorylation of IRS-1 at serine residues (IRS-1pSer), with a consequent loss of substrate affinity as observed in T2D. As reported by Bomfim et al. (2012), in the mouse hippocampal neurons $A \beta$ oligomers are also thought to activate the TNF- $\alpha / \mathrm{JNK}$ signaling, inducing insulin resistance (De Felice, 2013). The GLP-1RAs not only prevent JNK/IKK activation, but promote insulin activation by PI3K/AKT axis, with the subsequent activation of mTOR and the block of GSK$3 \beta$, an essential kinase also involved for the phosphorylation of tau protein (Moloney et al., 2010). Ma et al. (2015) reported that liraglutide administration prevented tau hyperphosphorylation associated with aging in diabetic $\mathrm{db} / \mathrm{db}$ mouse.

The role of vascular dysfunction has recently emerged as significant contributor in the pathophysiology of AD. Bloodbrain barrier and cerebral blood flow reduction might precede $\mathrm{A} \beta$ oligomers and tau deposition and it is associated to cognitive decline (Hachinski et al., 2019; Nation et al., 2019). In APP/PS1 transgenic mice liraglutide reduced the incidence of cerebral microanuerysms and leakage (Kelly et al., 2015).

Glucagon-like peptide-1 receptor agonists have shown neuroprotective effects in several preclinical studies in AD. Notably, they seem to improve nearly all neuropathological features in $\mathrm{AD}$ and cognitive functions as well. In 12-month-old female APP/PS1/tau AD mouse model, neurofibrillary tangles, amyloid plaques, and neuroinflammation in the hippocampi have been reduced by lixisenatide (Cai et al., 2018). In the rat model, lixisenatide also prevented synaptic damage induced by $\mathrm{A} \beta$ accumulation and strengthened spatial memory by affecting the PI3K-Akt-GSK3 $\beta$ (Cai et al., 2014). The GLP-1RA exenatide $(20 \mu \mathrm{gr} / \mathrm{kg} / \mathrm{day}$, intraperitoneally for 2 weeks) reduced neuroinflammation by suppressing the TNF- $\alpha$ levels in rats. Furthermore, it improved memory and prevented the loss of hippocampal neurons (Solmaz et al., 2015).

Recently, it has been observed that 4-week-treatment with exendin-4 reversed memory impairment in APP/PS1 mice, downregulating the aberrant $N$-acetylglucosaminyltransferase III expression through the Akt/GSK-3 $\beta / \beta$-catenin signaling pathway in neurons. $N$-Acetylglucosamine levels seem to be increased in the cerebrospinal fluid of most $\mathrm{AD}$ patients, and the levels of $N$-acetylglucosaminyltransferase III, a glycosyltransferase responsible for synthesizing a bisecting GlcNAc residue, were found to be highly expressed in the brains of AD patients as well (Wang et al., 2018).

Liraglutide (25 nmol/kg, intraperitoneally, for 2 months) improved spatial memory in 14-month-old APP/PS1 mouse model, compared to saline-treated mice. It also reduced inflammation and plaque load, while neuronal progenitor cell in the dentate gyrus increased. Long-term potentiation was significantly enhanced as well and synapse numbers increased in the hippocampus and cortex (McClean and Holscher, 2014). In another study, the same authors observed that liraglutide might also protect from progressive neurodegeneration that develops in AD: in 2-month old mice, liraglutide (once-daily intraperitoneally for 8 months) contrasted synaptic damage and improved memory. In addition, amyloid plaque load was reduced, inflammation was reduced in the cortex, and 
neurogenesis was enhanced in the dentate gyrus (McClean et al., 2015). On the contrary, other authors did not report beneficial effects of liraglutide on cerebral plaque load, in APP/PS1 transgenic mouse models of $\mathrm{AD}$ with two different clinical APP/PS1 mutations (Hansen et al., 2016). In the mouse model, memory deficit was improved by subcutaneous administration of liraglutide ( $25 \mathrm{nmol} /$ day once daily for 8 week), decreasing the phosphorylation of tau (Qi et al., 2016).

Furthermore, in APP/PS-1 mice at different ages, chronic administration of liraglutide promoted neural progenitor cells proliferation. Both acute and chronic treatment increased the number of immature neurons in animals at all ages, and the differentiation into mature neurons was observed for most immature cells (Parthsarathy and Holscher, 2013).

Even in a mouse model of pathological aging, which shares neurobehavioral and neuropathological dysfunction with sporadic AD at an early phase, liraglutide increased the number of CA1 pyramidal neuron in hippocampus and improved memory (Hansen et al., 2015).

The effects of GLP-1RAs on synaptic protection might involve the modulation of the brain-derived neurotrophic factor (BDNF), a trophic factor which promotes neural progenitor cell differentiation and survival. Indeed, exenatide activates the transcription factor CREB with an increase of BDNF protein expression promoting the activation of neurotrophic pathway and inhibiting apoptosis in a mouse model of age-dependent cognitive dysfunction, potentiating long-term memory (Bomba et al., 2018). Even in a mouse model of AD (the 3xTg-AD undergoing high fat diet), exenatide reverted the impairment of BDNF signaling and neuroinflammation (Bomba et al., 2019).

In the last few years, even dual and triple receptor agonists have been developed, with remarkable results in animal models. Indeed, GLP-1/gastric inhibitory polypeptide (GIP) dual agonist DA5-CH strengthened working memory and long-term spatial memory in APP/PS1 transgenic AD mouse model (9-month-old). It also led to a reduction in hippocampal amyloid senile plaques and in phosphorylated tau protein. The deficits in hippocampal late-phase long-term potentiation were reversed and $\mathrm{p}$-PI3K and $\mathrm{p}$-AKT growth factor kinases were up regulated. The excessive activation of p-GSK $\beta$ was prevented in the hippocampus (Cai et al., 2018).

\section{REFERENCES}

Aroda, V. R. (2018). A review of GLP-1 receptor agonists: evolution and advancement, through the lens of randomised controlled trials. Diabetes Obes. Metab. 20(Suppl. 1), 22-33. doi: 10.1111/dom. 13162

Athauda, D., and Foltynie, T. (2016). The glucagon-like peptide 1 (GLP) receptor as a therapeutic target in Parkinson's disease: mechanisms of action. Drug Discov. Today 21, 802-818. doi: 10.1016/j.drudis.2016.01.013

Athauda, D., Gulyani, S., Karnati, H. K., Li, Y., Tweedie, D., Mustapic, M., et al. (2019). Utility of neuronal-derived exosomes to examine molecular mechanisms that affect motor function in patients with parkinson disease: a secondary analysis of the exenatide-PD trial. JAMA Neurol. 76, 420-429. doi: 10.1001/jamaneurol.2018.4304

Athauda, D., Maclagan, K., Budnik, N., Zampedri, L., Hibbert, S., Skene, S. S., et al. (2018). What effects might exenatide have on non-motor symptoms in
Promising results have been observed with the dual GLP1/GIP receptor agonist DA-JC4 as well, which decreased phosphorylated tau levels in the rat cerebral cortex and hippocampus, prevented spatial learning dysfunction, attenuated chronic inflammation response in the brain, reduced apoptosis, and reactivated insulin signaling pathways in STZ-induced AD rat model (Shi et al., 2017). Recently, a triple receptor agonist, activating GLP-1, GIP, and glucagon receptors, rescued memory dysfunction, showed anti-apoptotic effects, enhanced synaptophysin, protected from synaptic loss, reduced the total amount of $\mathrm{A} \beta$, and reduced neuroinflammation (activated microglia and astrocytes) and oxidative stress in the cortex and hippocampus (Tai et al., 2018).

Despite the large amount of evidence about the neuroprotective effects of GLP-1RAs in animal models of AD, human studies are still scant. In a randomized, controlled, double-blind intervention study in $\mathrm{AD}$ patients, no effect on the deposition of $A \beta$ was observed in patients treated for 6 months with liraglutide, compared to placebo (Egefjord et al., 2012). In a more recent 26-week, double-blind RCT, although glucose metabolism increased in multiple regions in patients with $\mathrm{AD}$ treated with liraglutide compared to placebo, the statistical power of the study was insufficient to reach a conclusion about $A \beta$ load and cognition measures (Gejl et al., 2016).

\section{CONCLUSION}

Overall, the results on the effects of GLP-1RAs in animal models of neurodegenerative diseases are encouraging. However, further clinical research is needed to clarify whether they might be potential agents for the treatment of $\mathrm{PD}$ and $\mathrm{AD}$ and other forms of cognitive impairment.

\section{AUTHOR CONTRIBUTIONS}

MD'E and SM conceptualized and critically revised the manuscript. TF, MG, and MCG performed the PubMed search and wrote the mini-review. BM and AG critically revised the article for intellectual content.

parkinson's disease: a post hoc analysis. J. Parkinsons Dis. 8, 247-258. doi: 10.3233/JPD-181329

Athauda, D., Maclagan, K., Skene, S. S., Bajwa-Joseph, M., Letchford, D., Chowdhury, K., et al. (2017). Exenatide once weekly versus placebo in Parkinson's disease: a randomised, double-blind, placebo-controlled trial. Lancet 390, 1664-1675. doi: 10.1016/S0140-6736(17)31585-4

Badawi, G. A., Abd El Fattah, M. A., Zaki, H. F., and El Sayed, M. I. (2017). Sitagliptin and liraglutide reversed nigrostriatal degeneration of rodent brain in rotenone-induced Parkinson's disease. Inflammopharmacology 25, 369-382. doi: 10.1007/s10787-017-0331-6

Badawi, G. A., Abd El Fattah, M. A., Zaki, H. F., and El Sayed, M. I. (2019). Sitagliptin and liraglutide modulate L-dopa effect and attenuate dyskinetic movements in rotenone-lesioned rats. Neurotox. Res. 35, 635-653. doi: 10.1007/ s12640-019-9998-3

Bertilsson, G., Patrone, C., Zachrisson, O., Andersson, A., Dannaeus, K., Heidrich, J., et al. (2008). Peptide hormone exendin-4 stimulates subventricular zone 
neurogenesis in the adult rodent brain and induces recovery in an animal model of Parkinson's disease. J. Neurosci. Res. 86, 326-338. doi: 10.1002/jnr.21483

Bomba, M., Granzotto, A., Castelli, V., Massetti, N., Silvestri, E., Canzoniero, L. M. T., et al. (2018). Exenatide exerts cognitive effects by modulating the BDNF-TrkB neurotrophic axis in adult mice. Neurobiol. Aging 64, 33-43. doi: 10.1016/j.neurobiolaging.2017.12.009

Bomba, M., Granzotto, A., Castelli, V., Onofrj, M., Lattanzio, R., Cimini, A., et al. (2019). Exenatide reverts the high-fat-diet-induced impairment of BDNF signaling and inflammatory response in an animal model of Alzheimer's disease. J. Alzheimers Dis. 70, 793-810. doi: 10.3233/JAD- 190237

Bomfim, T. R., Forny-Germano, L., Sathler, L. B., Brito-Moreira, J., Houzel, J. C., Decker, H., et al. (2012). An anti-diabetes agent protects the mouse brain from defective insulin signaling caused by Alzheimer's disease- associated Abeta oligomers. J. Clin. Invest. 122, 1339-1353. doi: 10.1172/JCI57256

Cabou, C., and Burcelin, R. (2011). GLP-1, the gut-brain, and brain-periphery axes. Rev. Diabet. Stud. 8, 418-431. doi: 10.1900/RDS.2011.8.418

Cai, H. Y., Holscher, C., Yue, X. H., Zhang, S. X., Wang, X. H., Qiao, F., et al. (2014). Lixisenatide rescues spatial memory and synaptic plasticity from amyloid beta protein-induced impairments in rats. Neuroscience 277, 6-13. doi: 10.1016/j. neuroscience.2014.02.022

Cai, H. Y., Yang, J. T., Wang, Z. J., Zhang, J., Yang, W., Wu, M. N., et al. (2018). Lixisenatide reduces amyloid plaques, neurofibrillary tangles and neuroinflammation in an APP/PS1/tau mouse model of Alzheimer's disease. Biochem. Biophys. Res. Commun. 495, 1034-1040. doi: 10.1016/j.bbrc.2017.11. 114

Calsolaro, V., and Edison, P. (2015). Novel GLP-1 (Glucagon-Like Peptide-1) analogues and insulin in the treatment for Alzheimer's disease and other neurodegenerative diseases. CNS Drugs 29, 1023-1039. doi: 10.1007/s40263015-0301-8

De Felice, F. G. (2013). Alzheimer's disease and insulin resistance: translating basic science into clinical applications. J. Clin. Invest. 123, 531-539. doi: 10.1172/ JCI64595

Decarie-Spain, L., Fisette, A., Zhu, Z., Yang, B., DiMarchi, R. D., Tschop, M. H., et al. (2019). GLP-1/dexamethasone inhibits food reward without inducing mood and memory deficits in mice. Neuropharmacology 151, 55-63. doi: 10. 1016/j.neuropharm.2019.03.035

Egefjord, L., Gejl, M., Moller, A., Braendgaard, H., Gottrup, H., Antropova, O., et al. (2012). Effects of liraglutide on neurodegeneration, blood flow and cognition in Alzheimer s disease - protocol for a controlled, randomized double-blinded trial. Dan. Med. J. 59:A4519.

Fang, Y., Jiang, D., Wang, Y., Wang, Q., Lv, D., Liu, J., et al. (2018). Neuroprotection of rhGLP-1 in diabetic rats with cerebral ischemia/reperfusion injury via regulation of oxidative stress, EAAT2, and apoptosis. Drug Dev. Res. 79, 249 259. doi: $10.1002 /$ ddr.21439

Farilla, L., Bulotta, A., Hirshberg, B., Li Calzi, S., Khoury, N., Noushmehr, H., et al. (2003). Glucagon-like peptide 1 inhibits cell apoptosis and improves glucose responsiveness of freshly isolated human islets. Endocrinology 144, 5149-5158. doi: 10.1210/en.2003-323

Filchenko, I., Simanenkova, A., Chefu, S., Kolpakova, M., and Vlasov, T. (2018). Neuroprotective effect of glucagon-like peptide-1 receptor agonist is independent of glycaemia normalization in type two diabetic rats. Diab. Vasc. Dis. Res. 15, 567-570. doi: 10.1177/1479164118788079

Fiory, F., Perruolo, G., Cimmino, I., Cabaro, S., Pignalosa, F. C., Miele, C., et al. (2019). The relevance of insulin action in the dopaminergic system. Front. Neurosci. 13:868. doi: 10.3389/fnins.2019.00868

Freude, S., Plum, L., Schnitker, J., Leeser, U., Udelhoven, M., Krone, W., et al. (2005). Peripheral hyperinsulinemia promotes tau phosphorylation in vivo. Diabetes 54, 3343-3348. doi: 10.2337/diabetes.54.12.3343

Gault, V. A., and Holscher, C. (2008). GLP-1 agonists facilitate hippocampal LTP and reverse the impairment of LTP induced by beta-amyloid. Eur. J. Pharmacol. 587, 112-117. doi: 10.1016/j.ejphar.2008.03.025

Gejl, M., Gjedde, A., Egefjord, L., Moller, A., Hansen, S. B., Vang, K., et al. (2016). In Alzheimer's disease, 6-month treatment with glp-1 analog prevents decline of brain glucose metabolism: randomized, placebo-controlled, double-blind clinical trial. Front. Aging Neurosci. 8:108. doi: 10.3389/fnagi.2016.00108

Gomez-Peralta, F., and Abreu, C. (2019). Profile of semaglutide in the management of type 2 diabetes: design, development, and place in therapy. Drug Des. Dev. Ther. 13, 731-738. doi: 10.2147/DDDT.S165372
Haas, C. B., Kalinine, E., Zimmer, E. R., Hansel, G., Brochier, A. W., Oses, J. P., et al. (2016). Brain insulin administration triggers distinct cognitive and neurotrophic responses in young and aged rats. Mol. Neurobiol. 53, 5807-5817. doi: 10.1007/s12035-015-9494-6

Hachinski, V., Einhaupl, K., Ganten, D., Alladi, S., Brayne, C., Stephan, B. C. M., et al. (2019). Preventing dementia by preventing stroke: the Berlin Manifesto. Alzheimers Dement. 15, 961-984. doi: 10.1016/j.jalz.2019. 06.001

Hansen, H. H., Fabricius, K., Barkholt, P., Kongsbak-Wismann, P., Schlumberger, C., Jelsing, J., et al. (2016). Long-term treatment with liraglutide, a glucagonlike peptide-1 (GLP-1) receptor agonist, has no effect on beta-amyloid plaque load in two transgenic APP/PS1 mouse models of Alzheimer's disease. PLoS One 11:e0158205. doi: 10.1371/journal.pone.0158205

Hansen, H. H., Fabricius, K., Barkholt, P., Niehoff, M. L., Morley, J. E., Jelsing, J., et al. (2015). The GLP-1 receptor agonist liraglutide improves memory function and increases hippocampal CA1 neuronal numbers in a senescenceaccelerated mouse model of Alzheimer's disease. J. Alzheimers Dis. 46, 877-888. doi: 10.3233/JAD- 143090

Harkavyi, A., Abuirmeileh, A., Lever, R., Kingsbury, A. E., Biggs, C. S., and Whitton, P. S. (2008). Glucagon-like peptide 1 receptor stimulation reverses key deficits in distinct rodent models of Parkinson's disease. J. Neuroinflammation 5:19. doi: 10.1186/1742-2094-5-19

Hopsu-Havu, V. K., and Glenner, G. G. (1966). A new dipeptide naphthylamidase hydrolyzing glycyl-prolyl-beta-naphthylamide. Histochemie 7, 197-201. doi: 10.1007/bf00577838

Kalia, L. V., and Lang, A. E. (2015). Parkinson's disease. Lancet 386, 896-912. doi: 10.1016/S0140-6736(14)61393-3

Katsurada, K., and Yada, T. (2016). Neural effects of gut- and brain-derived glucagon-like peptide-1 and its receptor agonist. J. Diabetes Investig. 7(Suppl. 1), 64-69. doi: $10.1111 /$ jdi.12464

Kelly, P., McClean, P. L., Ackermann, M., Konerding, M. A., Holscher, C., and Mitchell, C. A. (2015). Restoration of cerebral and systemic microvascular architecture in APP/PS1 transgenic mice following treatment with Liraglutide. Microcirculation 22, 133-145. doi: 10.1111/micc.12186

Kinzig, K. P., D’Alessio, D. A., Herman, J. P., Sakai, R. R., Vahl, T. P., Figueiredo, H. F., et al. (2003). CNS glucagon-like peptide-1 receptors mediate endocrine and anxiety responses to interoceptive and psychogenic stressors. J. Neurosci. 23, 6163-6170. doi: 10.1523/jneurosci.23-15-06163.2003

Koekkoek, P. S., Kappelle, L. J., van den Berg, E., Rutten, G. E., and Biessels, G. J. (2015). Cognitive function in patients with diabetes mellitus: guidance for daily care. Lancet Neurol. 14, 329-340. doi: 10.1016/S1474-4422(14)70249-2

Kong, F. J., Wu, J. H., Sun, S. Y., Ma, L. L., and Zhou, J. Q. (2018). Liraglutide ameliorates cognitive decline by promoting autophagy via the AMP-activated protein kinase/mammalian target of rapamycin pathway in a streptozotocininduced mouse model of diabetes. Neuropharmacology 131, 316-325. doi: 10 . 1016/j.neuropharm.2018.01.001

Lennox, R., Flatt, P. R., and Gault, V. A. (2014). Lixisenatide improves recognition memory and exerts neuroprotective actions in high-fat fed mice. Peptides 61, 38-47. doi: 10.1016/j.peptides.2014.08.014

Lesage, S., and Brice, A. (2009). Parkinson's disease: from monogenic forms to genetic susceptibility factors. Hum. Mol. Genet. 18, R48-R59. doi: 10.1093/hmg/ ddp012

Li, Y., Perry, T., Kindy, M. S., Harvey, B. K., Tweedie, D., Holloway, H. W., et al. (2009). GLP-1 receptor stimulation preserves primary cortical and dopaminergic neurons in cellular and rodent models of stroke and Parkinsonism. Proc. Natl. Acad. Sci. U.S.A. 106, 1285-1290. doi: 10.1073/pnas. 0806720106

Liu, W., Jalewa, J., Sharma, M., Li, G., Li, L., and Holscher, C. (2015). Neuroprotective effects of lixisenatide and liraglutide in the 1-methyl-4-phenyl1,2,3,6-tetrahydropyridine mouse model of Parkinson's disease. Neuroscience 303, 42-50. doi: 10.1016/j.neuroscience.2015.06.054

Ma, D., Liu, X., Liu, J., Li, M., Chen, L., Gao, M., et al. (2019). Long-term liraglutide ameliorates nigrostriatal impairment via regulating AMPK/PGC-1a signaling in diabetic mice. Brain Res. 1714, 126-132. doi: 10.1016/j.brainres.2019.02.030

Ma, D. L., Chen, F. Q., Xu, W. J., Yue, W. Z., Yuan, G., and Yang, Y. (2015). Early intervention with glucagon-like peptide 1 analog liraglutide prevents tau hyperphosphorylation in diabetic $\mathrm{db} / \mathrm{db}$ mice. J. Neurochem. 135, 301-308. doi: $10.1111 /$ jnc. 13248 
Masciopinto, F., Di Pietro, N., Corona, C., Bomba, M., Pipino, C., Curcio, M., et al. (2012). Effects of long-term treatment with pioglitazone on cognition and glucose metabolism of PS1-KI, 3xTg-AD, and wild-type mice. Cell Death Dis. 3:e448. doi: 10.1038/cddis.2012.189

McClean, P. L., and Holscher, C. (2014). Liraglutide can reverse memory impairment, synaptic loss and reduce plaque load in aged APP/PS1 mice, a model of Alzheimer's disease. Neuropharmacology 76(Pt A), 57-67. doi: 10. 1016/j.neuropharm.2013.08.005

McClean, P. L., Jalewa, J., and Holscher, C. (2015). Prophylactic liraglutide treatment prevents amyloid plaque deposition, chronic inflammation and memory impairment in APP/PS1 mice. Behav. Brain Res. 293, 96-106. doi: 10.1016/j.bbr.2015.07.024

Meier, J. J., Gallwitz, B., Schmidt, W. E., and Nauck, M. A. (2002). Glucagonlike peptide 1 as a regulator of food intake and body weight: therapeutic perspectives. Eur. J. Pharmacol. 440, 269-279. doi: 10.1016/s0014-2999(02) 01434-6

Meloni, A. R., DeYoung, M. B., Lowe, C., and Parkes, D. G. (2013). GLP-1 receptor activated insulin secretion from pancreatic beta-cells: mechanism and glucose dependence. Diabetes Obes. Metab. 15, 15-27. doi: 10.1111/j.1463-1326.2012. 01663.x

Moloney, A. M., Griffin, R. J., Timmons, S., O’Connor, R., Ravid, R., and O’Neill, C. (2010). Defects in IGF-1 receptor, insulin receptor and IRS-1/2 in Alzheimer's disease indicate possible resistance to IGF-1 and insulin signalling. Neurobiol. Aging 31, 224-243. doi: 10.1016/j.neurobiolaging.2008.04.002

Muscogiuri, G., DeFronzo, R. A., Gastaldelli, A., and Holst, J. J. (2017). Glucagonlike peptide- 1 and the central/peripheral nervous system: crosstalk in diabetes. Trends Endocrinol. Metab. 28, 88-103. doi: 10.1016/j.tem.2016.10.001

Nation, D. A., Sweeney, M. D., Montagne, A., Sagare, A. P., D’Orazio, L. M., Pachicano, M., et al. (2019). Blood-brain barrier breakdown is an early biomarker of human cognitive dysfunction. Nat. Med. 25, 270-276. doi: 10. 1038/s41591-018-0297-y

Nauck, M. A., Vardarli, I., Deacon, C. F., Holst, J. J., and Meier, J. J. (2011). Secretion of glucagon-like peptide-1 (GLP-1) in type 2 diabetes: what is up, what is down? Diabetologia 54, 10-18. doi: 10.1007/s00125-010-1896-4

Olanow, C. W., and Tatton, W. G. (1999). Etiology and pathogenesis of Parkinson's disease. Annu. Rev. Neurosci. 22, 123-144. doi: 10.1146/annurev.neuro.22.1. 123

Palleria, C., Leo, A., Andreozzi, F., Citraro, R., Iannone, M., Spiga, R., et al. (2017). Liraglutide prevents cognitive decline in a rat model of streptozotocin-induced diabetes independently from its peripheral metabolic effects. Behav. Brain Res. 321, 157-169. doi: 10.1016/j.bbr.2017.01.004

Parthsarathy, V., and Holscher, C. (2013). Chronic treatment with the GLP1 analogue liraglutide increases cell proliferation and differentiation into neurons in an AD mouse model. PLoS One 8:e58784. doi: 10.1371/journal.pone.0058784

Pozo, L., Bello, F., Suarez, A., Ochoa-Martinez, F. E., Mendez, Y., Chang, C. H., et al. (2019). Novel pharmacological therapy in type 2 diabetes mellitus with established cardiovascular disease: current evidence. World J. Diabetes 10, 291-303. doi: 10.4239/wjd.v10.i5.291

Qi, L., Ke, L., Liu, X., Liao, L., Ke, S., Liu, X., et al. (2016). Subcutaneous administration of liraglutide ameliorates learning and memory impairment by modulating tau hyperphosphorylation via the glycogen synthase kinase-3beta pathway in an amyloid beta protein induced alzheimer disease mouse model. Eur. J. Pharmacol. 783, 23-32. doi: 10.1016/j.ejphar.2016.04.052

Ruttimann, E. B., Arnold, M., Hillebrand, J. J., Geary, N., and Langhans, W. (2009). Intrameal hepatic portal and intraperitoneal infusions of glucagon-like peptide-1 reduce spontaneous meal size in the rat via different mechanisms. Endocrinology 150, 1174-1181. doi: 10.1210/en.2008-1221

Sandoval, D. A., and D'Alessio, D. A. (2015). Physiology of proglucagon peptides: role of glucagon and GLP-1 in health and disease. Physiol. Rev. 95, 513-548. doi: 10.1152/physrev.00013.2014

Shi, L., Zhang, Z., Li, L., and Holscher, C. (2017). A novel dual GLP-1/GIP receptor agonist alleviates cognitive decline by re-sensitizing insulin signaling in the
Alzheimer icv. STZ rat model. Behav. Brain Res. 327, 65-74. doi: 10.1016/j.bbr. 2017.03.032

Smith, N. K., Hackett, T. A., Galli, A., and Flynn, C. R. (2019). GLP-1: molecular mechanisms and outcomes of a complex signaling system. Neurochem. Int. 128, 94-105. doi: 10.1016/j.neuint.2019.04.010

Solmaz, V., Cinar, B. P., Yigitturk, G., Cavusoglu, T., Taskiran, D., and Erbas, O. (2015). Exenatide reduces TNF-alpha expression and improves hippocampal neuron numbers and memory in streptozotocin treated rats. Eur. J. Pharmacol. 765, 482-487. doi: 10.1016/j.ejphar.2015.09.024

Song, W. J., Seshadri, M., Ashraf, U., Mdluli, T., Mondal, P., Keil, M., et al. (2011). Snapin mediates incretin action and augments glucosedependent insulin secretion. Cell Metab. 13, 308-319. doi: 10.1016/j.cmet.2011. 02.002

Tai, J., Liu, W., Li, Y., Li, L., and Holscher, C. (2018). Neuroprotective effects of a triple GLP-1/GIP/glucagon receptor agonist in the APP/PS1 transgenic mouse model of Alzheimer's disease. Brain Res. 1678, 64-74. doi: 10.1016/j.brainres. 2017.10.012

Tramutola, A., Arena, A., Cini, C., Butterfield, D. A., and Barone, E. (2017). Modulation of GLP-1 signaling as a novel therapeutic approach in the treatment of Alzheimer's disease pathology. Expert Rev. Neurother. 17, 59-75. doi: 10. 1080/14737175.2017.1246183

van Dijk, G., and Thiele, T. E. (1999). Glucagon-like peptide-1 (7-36) amide: a central regulator of satiety and interoceptive stress. Neuropeptides 33, 406-414. doi: 10.1054/npep.1999.0053

Vandal, M., White, P. J., Tremblay, C., St-Amour, I., Chevrier, G., Emond, V., et al. (2014). Insulin reverses the high-fat diet-induced increase in brain Abeta and improves memory in an animal model of Alzheimer disease. Diabetes 63, 4291-4301. doi: 10.2337/db14-0375

Wang, Y., Chen, S., Xu, Z., Chen, S., Yao, W., and Gao, X. (2018). GLP-1 receptor agonists downregulate aberrant GnT-III expression in Alzheimer's disease models through the Akt/GSK-3beta/beta-catenin signaling. Neuropharmacology 131, 190-199. doi: 10.1016/j.neuropharm.2017. 11.048

World Health Organization (2017). Dementia Fact Sheets [Online]. Available at: https://www.who.int/en/news-room/fact-sheets/detail/dementia (accessed June 11, 2019).

Yang, Y., Fang, H., Xu, G., Zhen, Y., Zhang, Y., Tian, J., et al. (2018). Liraglutide improves cognitive impairment via the AMPK and PI3K/Akt signaling pathways in type 2 diabetic rats. Mol. Med. Rep. 18, 2449-2457. doi: 10.3892/ mmr.2018.9180

Yildirim Simsir, I., Soyaltin, U. E., and Cetinkalp, S. (2018). Glucagon like peptide1 (GLP-1) likes Alzheimer's disease. Diabetes Metab. Syndr. 12, 469-475. doi: 10.1016/j.dsx.2018.03.002

Zhang, L., Zhang, L., Li, L., and Holscher, C. (2018). Neuroprotective effects of the novel GLP-1 long acting analogue semaglutide in the MPTP Parkinson's disease mouse model. Neuropeptides 71, 70-80. doi: 10.1016/j.npep.2018.07.003

Zhang, L., Zhang, L., Li, L., and Holscher, C. (2019). Semaglutide is neuroprotective and reduces alpha-synuclein levels in the chronic MPTP mouse model of Parkinson's disease. J. Parkinsons Dis. 9, 157-171. doi: 10.3233/JPD-18 1503

Conflict of Interest: The authors declare that the research was conducted in the absence of any commercial or financial relationships that could be construed as a potential conflict of interest.

Copyright () 2019 Grieco, Giorgi, Gentile, d'Erme, Morano, Maras and Filardi. This is an open-access article distributed under the terms of the Creative Commons Attribution License (CC BY). The use, distribution or reproduction in other forums is permitted, provided the original author(s) and the copyright owner(s) are credited and that the original publication in this journal is cited, in accordance with accepted academic practice. No use, distribution or reproduction is permitted which does not comply with these terms. 